Disclosure of Interest: None declared

DOI: 10.1136/annrheumdis-2017-eular.4226

\section{SAT0075 DOES EARLY REMISSION LEAD TO BETTER 5-YEAR OUTCOMES THAN LOW DISEASE ACTIVITY? RESULTS FROM THE REAL LIFE NOR-DMARD STUDY}

V. Norvang, I.C. Olsen, E. Lie, E.K. Kristianslund, T.K. Kvien, E.A. Haavardsholm, T. Uhlig. Department of Rheumatology, Diakonhjemmet Hospital, Oslo, Norway

Background: When initiating therapy with synthetic disease-modifying antirheumatic drugs (sDMARDs) in patients with rheumatoid arthritis (RA), the recommended target is remission or low disease activity (LDA). Limited data exist on the impacts of reaching remission rather than LDA on long-term outcomes. Objectives: To compare RA-patients who achieved Simplified Disease Activity Index (SDAI) remission versus LDA 6 months after initiating SDMARD therapy, with regard to physical function, Health Related Quality of Life (HRQoL) and disease activity during 5 years of follow-up in a routine clinical setting.

Methods: Data were provided NOR-DMARD, a prospective multicentre longitudinal observational study. We selected DMARD-naïve patients with RA enrolled between December 2000 and April 2009 who had a registered visit with available SDAI status 6 months after initiating SDMARD therapy. Data on each patient were collected at baseline, after 3, 6 and 12 months, and yearly thereafter, including the modified Health Assessment Questionnaire (MHAQ), the Medical Outcomes Study 36-item Short-Form Health Survey (SF-36) with Physical and Mental Components Summary scores (PCS and MCS, respectively) and SF-6D, and assessments that allowed the calculation of the composite disease activity scores SDAI, Clinical Disease Activity Index (CDAI) and the Disease Activity Score based on 28 joint counts (DAS28). Multivariate linear mixed models were used to explore the effect of SDAI status at 6 months on physical function (MHAQ), HRQoL (SF-36 PCS and MCS, SF-6D) and disease activity (SDAI, CDAI, DAS28) during 5 year follow-up. The statistical models were adjusted for age, gender, disease duration and baseline disease activity. Furthermore, we performed mixed model analyses separately for patients in LDA, MDA and HDA at baseline, exploring the impact of SDAI status at 6 months on long-term disease activity in each sub-group.

Results: Of 1148 eligible patients, 867 patients (75.5\%) started with methotrexate in monotherapy and $281(24.5 \%)$ started with another sDMARD or sDMARD combination. Patients in SDAI remission $(n=145 ; 16.6 \%)$ rather than LDA $(n=454$; $39.5 \%) 6$ months after initiating therapy had better physical function (MHAQ, estimated mean difference $0.11-0.20, \mathrm{p}<0.02)$, higher SF-36 PCS $(4.13-8.16$, $\mathrm{p}<0.003)$ and SF-6D $(0.06-0.12, \mathrm{p}<0.0001)$, and lower disease activity (SDAI, $2.24-5.15, p<0.05$ ) for all visits during 5 years of follow-up. Stratified mixed models analyses of patients in SDAI LDA, MDA and HDA at baseline, resulted in an overall significant long-term beneficial effect of achieving remission rather than LDA at 6 months; however, the differences were less distinct for patients who were already in a state of LDA at baseline.

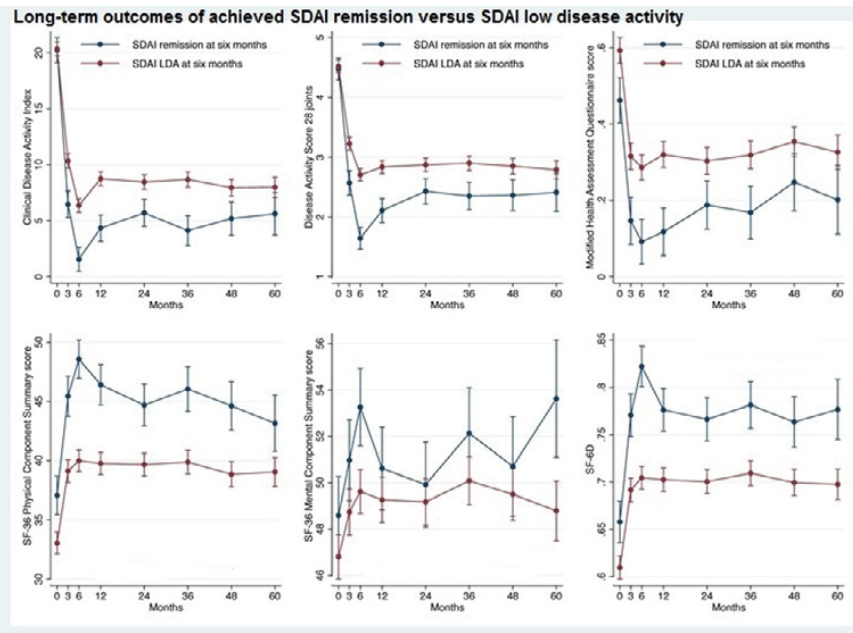

Conclusions: The achievement of SDAI remission 6 months after initiating DMARD-therapy was associated with favourable long-term outcomes compared with the achievement of SDAI low disease activity. The results from the study support that stringent remission is the optimal treatment target in patients with RA.

Disclosure of Interest: V. Norvang: None declared, I. Olsen: None declared, E. Lie Consultant for: AbbVie, Celgene, Hospira, Pfizer, UCB, E. Kristianslund: None declared, T. Kvien Consultant for: AbbVie, Biogen, BMS, Boehringer, Ingelheim, Celltrion, Eli Lilly, Epirus, Janssen, Merck-Serono, MSD, Mundipharma, Novartis, Oktal, Orion Pharma, Hospira/Pfizer, Roche, Sandoz, UCB, E. Haavardsholm Grant/research support from: AbbVie, Pfizer, Roche, MSD, UCB, Consultant for: AbbVie, Pfizer, Roche, Eli Lilly, Celgene, UCB, T. Uhlig: None declared DOI: 10.1136/annrheumdis-2017-eular.3874

\section{SAT0076 SERUM LEVEL OF SYNDECAN-4 AND ITS CORRELATION WITH CLINICAL PARAMETERS IN RHEUMATOID ARTHRITIS PATIENTS}

X. Ye, J. Zhao, Z. Zhang. Rheumatology and Clinical Immunology Department, Peking University First Hospital, Beijing, China

Background: Heparan sulfate proteoglycan syndecan-4 plays an important role in inflammation. However, the role of syndecan-4 in rheumatoid arthritis (RA) has not yet been elucidated.

Objectives: To detect serum level of syndecan-4 in RA patients and investigate its correlation with RA clinical parameters.

Methods: The concentration of serum syndecan-4 was assayed by enzyme-linked immunosorbent assay (ELISA). 43 patients' serum samples from our RA cohort study between 2014 and 2016, and 20 age- and gender-matched osteoarthritis (OA) patients' serum samples were collected and analyzed. Compared the serum syndecan- 4 levels in RA patients with DAS $28 \geq 3.2$ and DAS $28<3.2$ by Wilcoxon signed rank test. The relationships between serum syndecan-4 levels and RA clinical parameters (DAS28, rheumatoid factor (RF), erythrocyte sedimentation rate, C-reactive protein, etc.) were analyzed.

Results: Baseline serum syndecan-4 levels of RA patients were significantly higher than the matched OA patients $(1101.56 \mathrm{pg} / \mathrm{mL}$ vs $281.41 \mathrm{pg} / \mathrm{mL}, \mathrm{p}<0.001)$. In RA patients who had sera both at the point of DAS28 $\geq 3.2$ and DAS $28<3.2$ $(n=13)$, we found that the former syndecan-4 levels were higher than the latter (1666.22 $\mathrm{pg} / \mathrm{mL}$ vs $1378.34 \mathrm{pg} / \mathrm{mL}, \mathrm{p}=0.65$ ). The levels of serum syndecan-4 and RF were significantly and positively correlated in RA patients $(r=0.696, p=0.008)$. Furthermore, there is a tendency that serum syndecan-4 levels were higher in the RF-positive $(n=31)$ than in the RF-negative $(n=12) R A$ patients $(1344.43 \mathrm{pg} / \mathrm{mL}$ vs $971.27 \mathrm{pg} / \mathrm{mL}, \mathrm{p}=0.078$ )
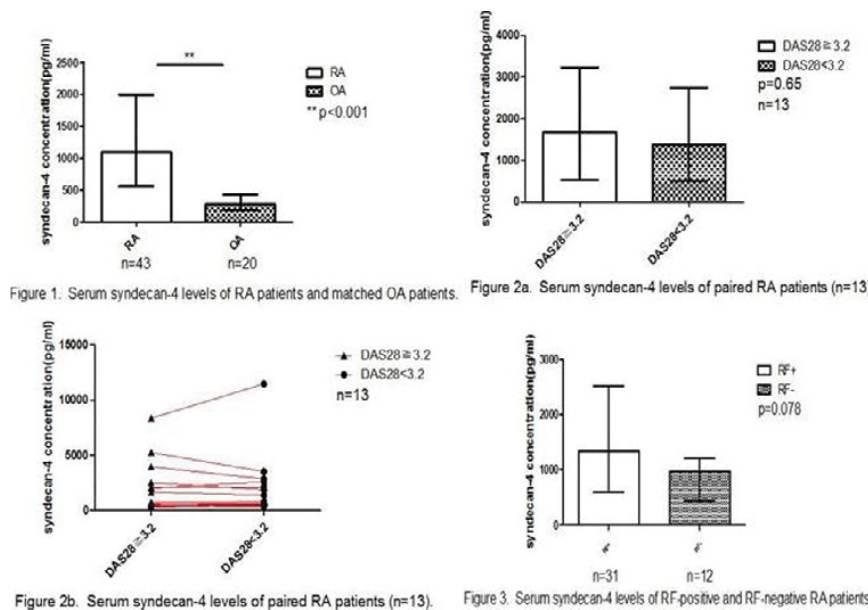

Conclusions: Compared with age- and gender- matched OA patients, serum syndecan-4 concentration is significantly higher in RA patients. Serum syndecan-4 level is positively correlated with RF. Syndecan-4 may play an important role in thes pathogenesis of RA. Further investigation is required to study the mechanism of syndecan-4 in RA.

References:

[1] Zhao J, Guo J, Wang L, et al. The role of a proliferation-inducing ligand (APRIL) in the pathogenesis of rheumatoid arthritis. Scand J Rheumatol 2014;43(6):462-9

[2] Patterson AM, Cartwright A, David G, et al. Differential expression of syndecans and glypicans in chronically inflamed synovium. Ann Rheum Dis 2008 May;67(5):592-601.

[3] Endo T, Ito K, Morimoto J, et al. Syndecan 4 Regulation of the Development of Autoimmune Arthritis in Mice by Modulating B Cell Migration and Germinal Center Formation. Arthritis Rheumatol 2015 Sep;67(9):2512-22.

Acknowledgements: We are indebted to all the patients who kindly participated in this study. This study was partly supported by Peking University Clinical Research Institute.

Disclosure of Interest: None declared

DOI: 10.1136/annrheumdis-2017-eular.1091

\section{SAT0077 CLINICAL FEATURES AND PERFORIN A91V GENE ANALYSIS IN SO-JIA CHILDREN WITH MACROPHAGE ACTIVATION SYNDROME}

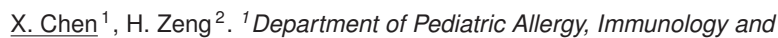
Rheumatolog; ${ }^{2}$ Department of Pediatric Allergy, Immunology and Rheumatology, Guangzhou Women and Children's Medical Center, Guangzhou, China

Objectives: Macrophage activation syndrome (MAS) is a severe, potentially life-threatening syndrome.Here we aim to review the precipitating events,clinical features, treatment, outcome and perforin A91V gene analysis in systemic onset juvenile idiopathic arthritis (SoJIA) children with MAS. 
Methods: Retrospective review of cases of MAS from a collected database of fourteen children with SoJIA from 2003 to 2008. Gene-specific polymerase chain reaction (PCR) primers were used to analyze the perforin A91V gene polymorphism.

Results: Fourteen patients (nine boys) were considered to have evidence of MAS, with age ranged from 4 months to 12 years. The primary diagnosis was systemic onset juvenile idiopathic arthritis. No medication was identified as trigger. Eleven had infections prior to MAS,specific infectious agents were identified in four. High fever, new onset hepatosplenomegaly, lymphadenopathy, liver dysfunction,abnormal lipid metabolism and hemophagocytosis were common clinical features.Two cases were with acute respiratory distress syndrome (ARDS), multiple organ failure (MOF) in three and three died. The perforin A91V (NCBI:SNP rs35947132) variant gene was detected in seven systemic onset juvenile idiopathic arthritis compolicated with MAS cases, but no mutation were found. Glucocorticoid, intravenous immunoglobulin, immunoimpressive therapy were effective and HP (Plasmapheresis) used in one serious case was also effective. Conclusions: MAS is a rare and potentially fatal complication of childhood rheumatoid diseases,especially systemic onset juvenile idiopathic arthritis. Most of our patients were male, and most cases were preceded by infection. Bone marrow studies support the diagnosis. MOF may be a poor prognostic sign.Aggressive early therapy is essential.

Disclosure of Interest: None declared

DOI: 10.1136/annrheumdis-2017-eular.1989

\section{SAT0078 MAGNETIC RESONANCE IMAGING OF THE HANDS IN} RHEUMATOID ARTHRITIS: UNILATERAL OR BILATERAL?

Y.-Q. Mo ${ }^{1}$, Z.-H. Yang ${ }^{2}$, H.-N. He ${ }^{3}$, J.-D. Ma ${ }^{1}$, J.-J. Liang ${ }^{1}$, L. Dai ${ }^{1}$

${ }^{1}$ Rheumatology; ${ }^{2}$ Radiology, Sun Yat-sen Memorial Hospital, Sun Yat-sen University: ${ }^{3}$ Zhongshan School of Medicine, Guangzhou, China

Background: MRI had higher sensitivity of detecting inflammation than physical examination and higher sensitivity of detecting bone damage than X-rays. Physical examination and X-rays are usually performed on bilateral hands of RA patients, however, MRI evaluation of unilateral hand was recommended by OMERACT at the very beginning when RA MRI score (RAMRIS) was validated in the databases only consisting of images of dominant wrists and/or metacarpal phalangeal joints (MCPJs). Recently, high performance of whole-body MRI and multi-channel synergic coil enable simultaneous scan of bilateral hands.

Objectives: To explore the advantages of bilateral hands MRI on RA.

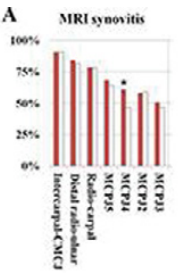

Wrigh
oleft
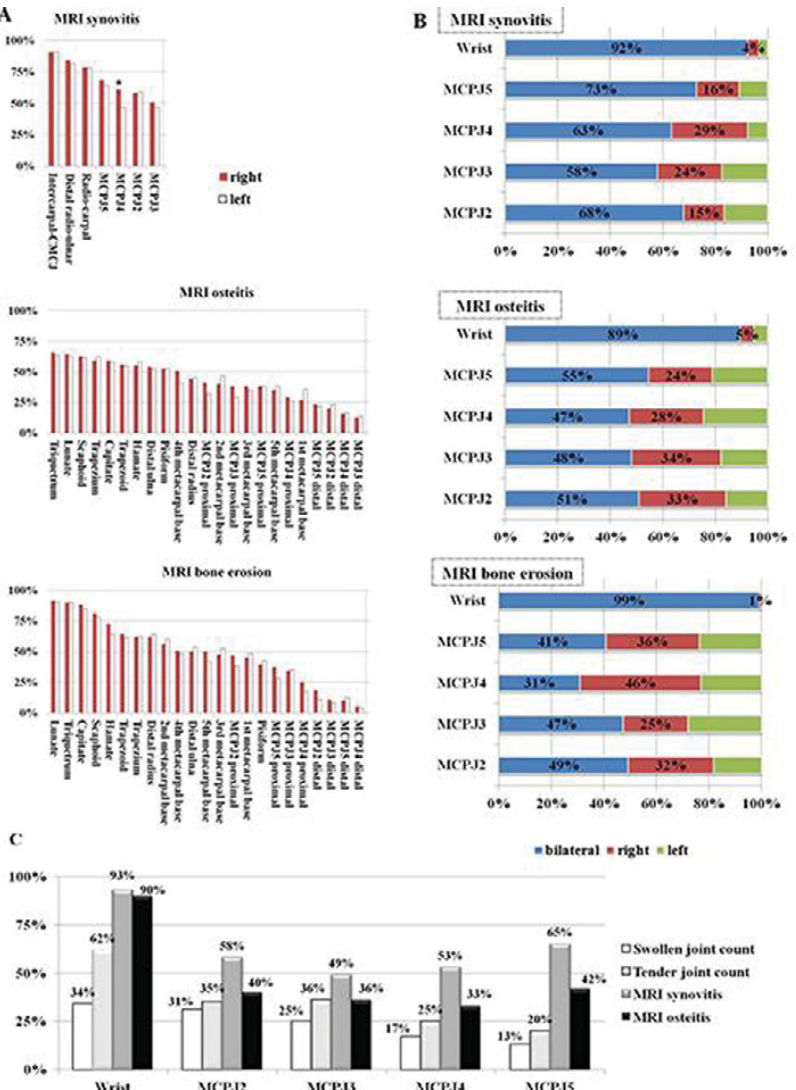

- bilateral = right = left
Methods: Consecutive hospitalized RA patients were recruited from April 2014 to April 2016 at Sun Yat-Sen Memorial Hospital, Sun Yat-Sen University. Together with assessing swelling and tenderness of 28 joints essential for DAS28, bilateral wrists and MCPJ2 5 of each patient were scanned simultaneously on 3.0T MRI system. MRI synovitis, osteitis and bone erosion were scored referred to the definitions and atlas of RAMRIS system.

Results: (1) Among 120 RA patients were included, $79 \%$ were female, age (median and IQR, similarly hereinafter) was $52(44 \sim 61)$ years, disease duration was 48 (12 120) months and DAS28-crp was 5.9 (4.7 6.9). The mean imaging time for the entire MRI examinations including the time of patient positioning and contrast agent injection was 23 minutes. The mean scoring time was 10 minutes for RAMRIS bilateral hands $_{\text {and } 7 \text { minutes for RAMRIS }}$ unilateral hand. Interreader ICC for

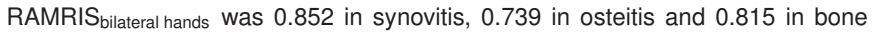
erosion.

(2) The frequencies of MRI synovitis, osteitis and bone erosion per joint or bone were shown in figure1A. For MCPJ2 5, 27\% 42\% of RA patients showed unilateral synovitis, $45 \% \sim 53 \%$ showed unilateral osteitis and $51 \% \sim 69 \%$ showed unilateral bone erosion (Figure1B).

(3) Of wrists with MRI synovitis, $73 \%$ were not swollen and $48 \%$ were not tender at physical examination. Of wrists with MRI osteitis, $74 \%$ were not swollen and $49 \%$ were not tender. Similar trend was seen in MCPJ2 5 (Figure1C).

(4) The most clinically involved hands (or dominant hand in case of equally severe involved) were usually chosen for MRI of unilateral hand. Here 70 patients showed more severe involvement in unilateral hand according to patients' complaint and physical examination. However, MRI verified $42 \%, 40 \%$ and $42 \%$ of them respectively had more synovits, osteitis and bone erosion in the opposite hand. The other 50 patients had equally severe involvement at physical examination and the dominant hands were chosen. MRI verified $30 \%, 49 \%$ and $33 \%$ of them respectively had more synovits, osteitis and bone erosion in the non-dominant hand.

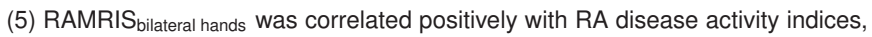
X-ray Sharp/vdH total score and subscores (all $p<0.05$ ). Moderate correlation was

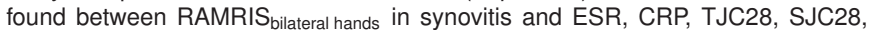
DAS28, SDAI or CDAI (all $r>0.4, p<0.001$ ). Relatively high correlation was found between RAMRIS bilateral hands in bone erosion and X-ray Sharp/vdH total score or erosion subscore (all $r>0.7, p<0.001$ ).

Conclusions: Performing MRI of bilateral hands yields additive information compared with imaging only unilateral hand.

Disclosure of Interest: None declared

DOI: 10.1136/annrheumdis-2017-eular.6106

\section{SAT0079 ASSOCIATION OF ALCOHOL CONSUMPTION AND DISEASE ACTIVITY IN JAPANESE PATIENTS WITH RHEUMATOID ARTHRITIS: ANALYSIS OF THE IORRA COHORT}

Y. Shimizu ${ }^{1}$, E. Tanaka ${ }^{1}$, E. Inoue ${ }^{1,2}$, M. Ochiai $^{1}$, R. Yamaguchi ${ }^{1}$, N. Sugimoto ${ }^{1}$ A. Nakajima ${ }^{1}$, K. kari $^{1}$, A. Taniguchi ${ }^{1}$, H. Yamanaka ${ }^{1}{ }^{1}$ Tokyo Women's Medical University Institute of Rheumatology; ${ }^{2}$ National Center for Child Health and Development Center for Clinical Research for Development, Tokyo, Japan

Background: While the results of several observational studies suggest that light-to-moderate alcohol consumption may decrease the risk for susceptibility to or severity of rheumatoid arthritis (RA) (1-3), findings regarding the effect of alcohol consumption on RA disease activity are conflicting. Furthermore, there are few reports of longitudinal studies regarding the effect of alcohol consumption on RA disease activity. In addition, although alcohol consumption in the Japanese general population was lower than that in European countries, according to the 2011 World Health Organization's Global Status Report on Alcohol and Health, there are few reports specifically concerning alcohol consumption in Japanese RA patients.

Objectives: To examine the longitudinal relationship between alcohol consumption and changes in disease activity in patients with RA using the Institute of Rheumatology, Rheumatoid Arthritis (IORRA) cohort database.

Methods: Subjects were RA patients who participated in the IORRA cohort study between October 2014 and October 2015. Patients were assigned to one of 5 groups according to alcohol-drinking status at baseline: the non-drinking group, drinking group 1 ( $0 \mathrm{~g}<$ amount of drinking per day [Alco-drink] $\leq 14 \mathrm{~g}$ ), drinking group 2 (14 g < Alco-drink $\leqq 28 \mathrm{~g})$, drinking group 3 (28 g $<$ Alco-drink $\leqq 50 \mathrm{~g})$, and drinking group 4 ( $50 \mathrm{~g}<$ Alco-drink). Multiple regression analyses were used to examine the relationship between alcohol consumption and baseline DAS28, and change in DAS28 between baseline and 1 year.

Results: Data from a total of 4,695 Japanese patients with RA (female: $86.6 \%$ mean age: 61.3 years old, mean disease duration: 15.2 years, and mean DAS28: $2.5)$ were analyzed. The number of patients and their characteristics (\% female mean age, mean disease duration, mean DAS28 at baseline/after 1 year) in the non-drinking group, and drinking groups 1, 2, 3, and 4 were 2,735 (92.8\%, 64.0 years old, 16.1 years, 2.7/2.7); 646 (89.9\%, 58.7 years old, 14.8 years, 2.4/2.5), $497(82.5 \%, 56.7$ years old, 13.8 years, 2.3/2.3), $444(71.6 \%, 56.4$ years old, 13.0 years, 2.3/2.3), and 373 (58.2\%, 57.5 years old, 13.7 years, $2.2 / 2.3)$, respectively. Baseline DAS28 in drinking groups $2(p=0.02), 3(p<0.01)$, and $4(p<0.01)$ was significantly lower than that in the nondrinking group. Multivariate regression analysis revealed that there was no association between alcohol-drinking status and the change in DAS28 at 1 year, after adjusting for DAS28 at baseline. 